This item was submitted to Loughborough's Research Repository by the author.

Items in Figshare are protected by copyright, with all rights reserved, unless otherwise indicated.

\title{
A study into refrigeration cycle working fluids using an air cycle machine environmental control system
}

PLEASE CITE THE PUBLISHED VERSION

http://dx.doi.org/10.2514/6.2016-2190

\section{PUBLISHER}

(c) American Institute of Aeronautics and Astronautics

\section{VERSION}

AM (Accepted Manuscript)

\section{PUBLISHER STATEMENT}

This work is made available according to the conditions of the Creative Commons Attribution-NonCommercialNoDerivatives 4.0 International (CC BY-NC-ND 4.0) licence. Full details of this licence are available at: https://creativecommons.org/licenses/by-nc-nd/4.0/

\section{LICENCE}

CC BY-NC-ND 4.0

\section{REPOSITORY RECORD}

Childs, Thomas, Andy Jones, Rui Chen, and Angus Murray. 2016. "A Study into Refrigeration Cycle Working Fluids Using an Air Cycle Machine Environmental Control System”. figshare. https://hdl.handle.net/2134/20140. 


\title{
A Study into Refrigeration Cycle Working Fluids using an Air Cycle Machine Environmental Control System
}

\author{
Thomas Childs ${ }^{1}$, Andy Jones ${ }^{1}$, Prof. Rui Chen ${ }^{1} *$ and Angus Murray ${ }^{2}$
}

\begin{abstract}
This study is the experimental analysis of a fast-jet military aircraft Environmental Control System (ECS) to the variation in Absolute Humidity (AH) of bleed air working fluid. A genuine fast-jet ECS operates within a ground test facility. The thermodynamic performance of the ECS is evaluated with two main metrics, Coefficient of Performance (CoP, a first law efficiency) and cooling capacity (function of exhaust temperature and mass flow rate). The ECS features Low Pressure Water Extraction (LPWE) with the use of a coalescing sock and centrifuge; the operation, efficiency and performance of this component are discussed in depth. The ECS inlet conditions (temperature, pressure and humidity) are typical of flight and atmospheric envelopes of the donor aircraft for all testing. A linear relationship is witnessed between increasing $\mathrm{AH}$ and decreasing $\mathrm{CoP}$, while the cooling capacity of the system exhibits a step change in performance based on induced phase change at the Cold Air Unit (CAU) turbine. The lack of visibility regarding working fluid phase change with traditional first law efficiency measures highlights the often misleading nature of this commonly used performance metric. While phase change is a fundamental requirement for water extraction, it is found to be thermodynamically expensive to system capability as the ECS has no mechanism to recover the energy released during the formation of condensate. This is typical of several complex system dynamics and thermodynamic trade-offs not apparent with dry working fluid. A number of time-dependent transient effects of water extractor coalescing sock blockage have been measured and discussed. The most extreme of these is the complete icing of this component causing a degradation in system performance and finally triggering the LPWE pressure release valve; replication of a typical operational problem. The difficulties of accurately modelling these behaviours is discussed and demonstrated to validate the experimental methodology utilised in this paper. It is concluded that an improved system performance is attainable through the accurate control of condensate generation and separation in the high pressure region of the CAU.
\end{abstract}

\section{Nomenclature}

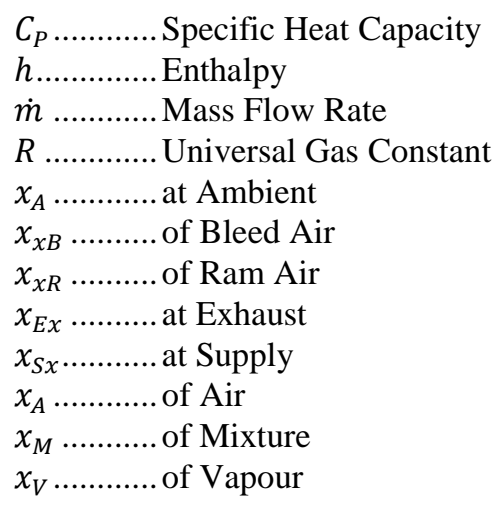

h...............Enthalpy

$\dot{m}$............. Mass Flow Rate

$R$............. Universal Gas Constant

$x_{A} \ldots \ldots . . . . .$. at Ambient

$x_{x B} \ldots \ldots . . . .$. of Bleed Air

$x_{x R} \ldots \ldots . . . .$. of Ram Air

$x_{E x} \ldots \ldots \ldots . .$. at Exhaust

$x_{S x} \ldots \ldots \ldots \ldots$ at Supply

$x_{A} \ldots \ldots \ldots \ldots$ of Air

$x_{V} \ldots \ldots \ldots . . . .$. of Vapour
$C_{P}$........... Specific Heat Capacity

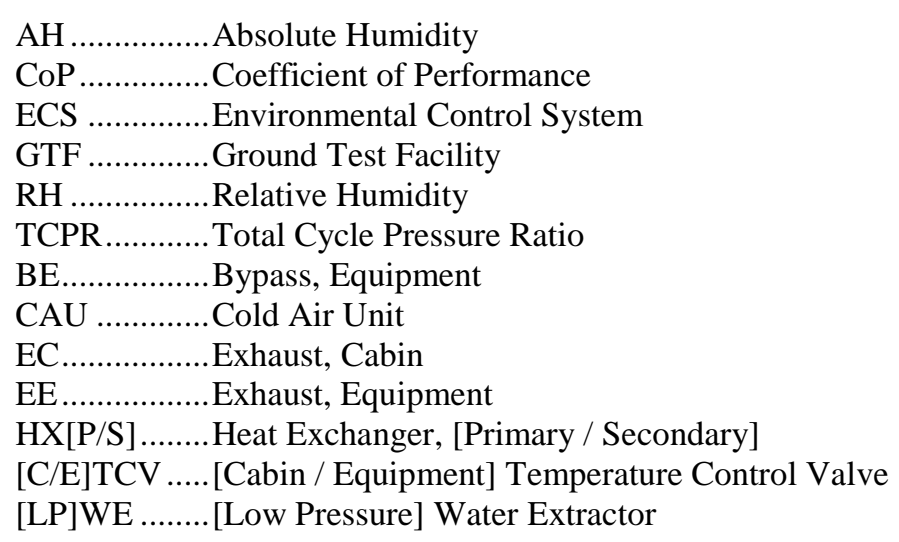

\footnotetext{
${ }^{1}$ Loughborough University, Leicestershire, UK

${ }^{2}$ BAE Systems Military Air \& Information, Warton, Lancashire, UK

* Correspondence address: R.Chen@lboro.ac.uk
} 


\section{Introduction}

$\mathrm{C}$ ONDENSATE control of cabin and equipment airflow for thermal management of a fast jet military aircraft is the responsibility of the Environmental Control System (ECS). There is no specific control of the Absolute Humidity $(\mathrm{AH})$ of the cabin and equipment air supply; however the requirement is to ensure that any water content remains in gaseous form. ${ }^{1}$ The ingress of free water in the cabin environment leads to increased risk of component corrosion, micro-organism growth and difficulty of effective canopy de-mist. Free water within the equipment airflow poses a serious risk to avionic component reliability and subsequently aircraft effectiveness. While the architecture of each avionic module is dependent on component power density, some modules do provide a direct contact between cooling airflow and active circuit cards. ${ }^{2}$

The ECS takes a feed of high pressure, high temperature engine bleed air as a working fluid, utilising ram air as a coolant. The absolute humidity of bleed air is dependent on geographical location and operational condition of the aircraft. Geographically, the worldwide variation in ambient humidity are detailed in Figure $1 .^{3}$ The most extreme humidity condition is defined as a 'Hot Humid' atmosphere and is typical of coastal regions of Saudi Arabia, Iran and Kuwait. Atmospheric conditions of up to $88 \%$ Relative Humidity (RH) at temperatures of up to $41^{\circ} \mathrm{C}$ generate $\mathrm{AH}$ as high as $47 \mathrm{gkg}-1$.

Operationally, humidity is only present at low altitudes. $\mathrm{RH}$ is a measure of the ability of air to hold moisture and is defined as the ratio of the partial pressure of water vapour to the saturation pressure of water at a given temperature. As the saturation pressure is dependent only on temperature and thermal lapse rate can be assumed linear within the troposphere (for a hot humid atmosphere), a direct relationship between altitude and humidity exists. As altitude increases, $\mathrm{RH}$ increases to the point at which partial pressure of water vapour is equal to the saturation pressure and water separates from the air $(100 \% \mathrm{RH})$. This is known as the dew point temperature and above this altitude, absolute humidity can be assumed negligible.

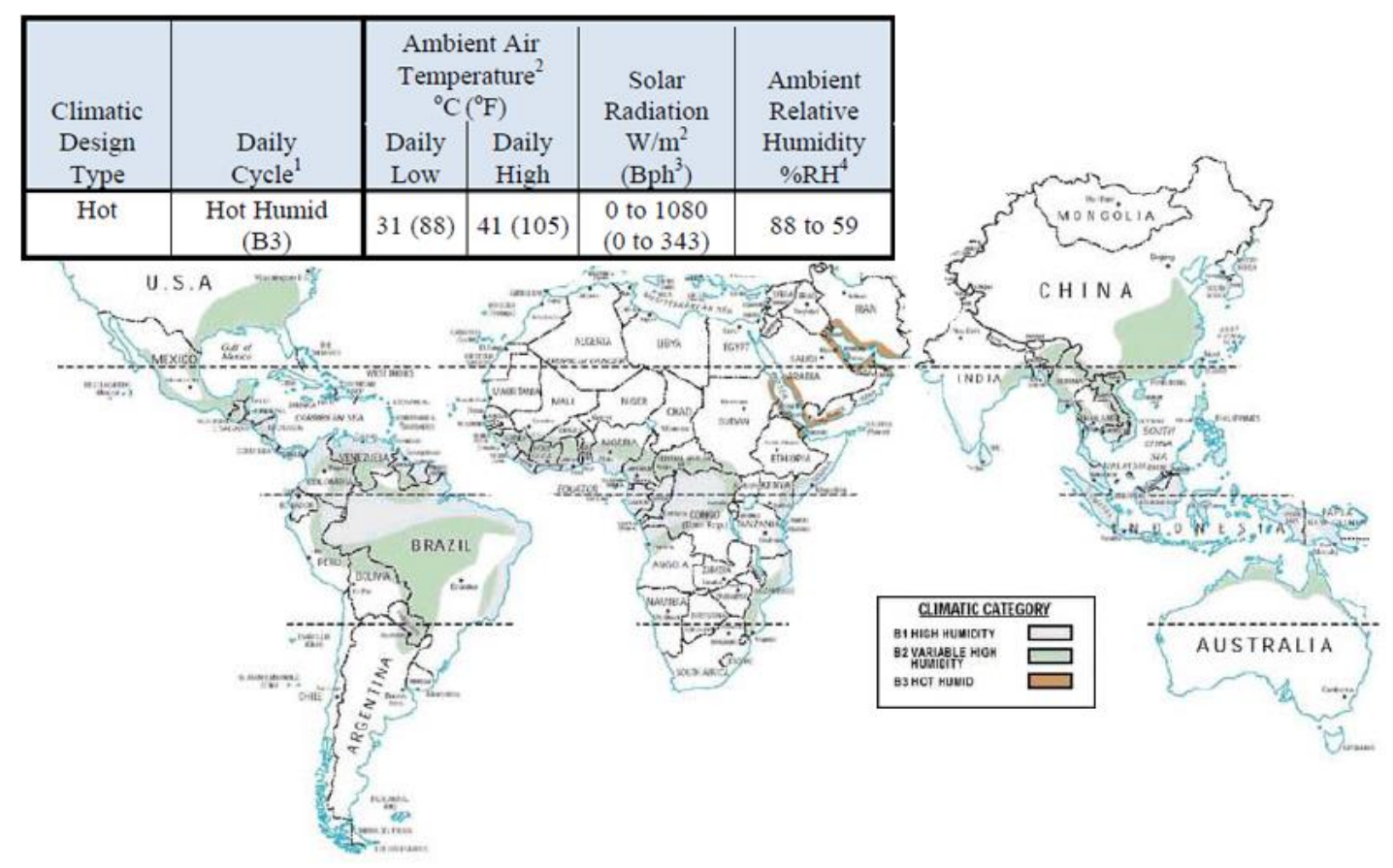

Figure 1. Definition of worldwide humidity regions. ${ }^{3}$

Uncontrolled condensate ingress to the cabin environment has been reported with bleed air driven Low Pressure Water Extraction (LPWE) ECS architectures, such as that displayed in Figure 2. A LPWE ECS utilises the expansion across the turbine to reduce the temperature of airflow below its dew point and generate condensate. The condensate is passed through a coalescing sock to form large droplets of water before a centrifugal removal process. $^{4}$

Typically (on operational aircraft) these cabin condensation ingress events are experienced in combination with high ECS exhaust temperatures, leading to difficulty in thermal management of distributed aircraft heat loads. This issue is often compounded by high solar and kinetic loading from the ambient environment and low ECS 
working fluid and coolant flow rates. ${ }^{5}$ This phenomenon is unique to high absolute humidity atmospheric conditions and therefore can be assumed to be a function of ECS working fluid.

The process of water extraction is well discussed in fast-jet ECS literature; however the accurate analysis of cabin condensate ingress from a LPWE ECS is relatively limited. ${ }^{1,46-8}$ As a result of the simple relationship between RH and airflow temperature, a one-dimensional modelling approach to condensation calculation for each component of the ECS is normally used for analysis. ${ }^{9}$ However, modelling is often inaccurate as the true process of condensation generation is a function of two-phase, three dimensional and transient flow conditions. The transient interactions of the water extraction process bring additional complexity in system dynamics and reduce the accuracy of one-dimensional saturation calculations.

The aim of this paper is to contribute to the knowledge gap of LPWE ECS response to the variation of inlet bleed air absolute moisture content. This experimental investigation utilises a genuine military fast-jet LPWE ECS operated within a Ground Test Facility (GTF). Accurate control of inlet temperature, pressure and absolute humidity conditions allow the assessment of the thermodynamic system response to replicated low altitude, high humidity operation. System performance is assessed both as a first-law cycle efficiency (Coefficient of Performance, CoP) and ability to generate cooling capability (pack exhaust temperature and mass flow rate). The generation and extraction of condensate is measured to evaluate the efficiency of the LPWE. Finally, the system response to a variation of bleed air working fluid is discussed in terms of real world performance of the aircraft thermal management capacity.

\section{Methodology}

The ECS used for this investigation is a simple two-wheel bootstrap cycle with LPWE. Two cycle bypass branches are taken upstream of the primary heat exchanger (HXP), regulated by butterfly valves, and reintroduced to the flow downstream of the turbine. A diagram of the system is shown in Figure 2, where numbered blocks represent instrumentation locations. The component naming convention gives the type and location in order: e.g. SB is Supply Bleed and ERS is Exhaust Ram Secondary. Temperature and pressure readings are taken at each location. Bleed air mass flow is measured at $\mathrm{SB}, \mathrm{EE}$, and $\mathrm{EC}$; whilst $\mathrm{BE}$ and $\mathrm{BC}$ are calibrated for mass flow against pressure drop and TCV position.

The first Temperature Control Valve (TCV) controls turbine-out temperature for application to forceconvection cooled avionics (equipment, ETCV, BE). Water extraction is then performed by a coalescing sock and centrifuge (WE). Finally the second TCV (cabin, CTCV, BC) bypass branch is reintroduced to the flow which is then distributed around the two-man cockpit and three avionics bays.

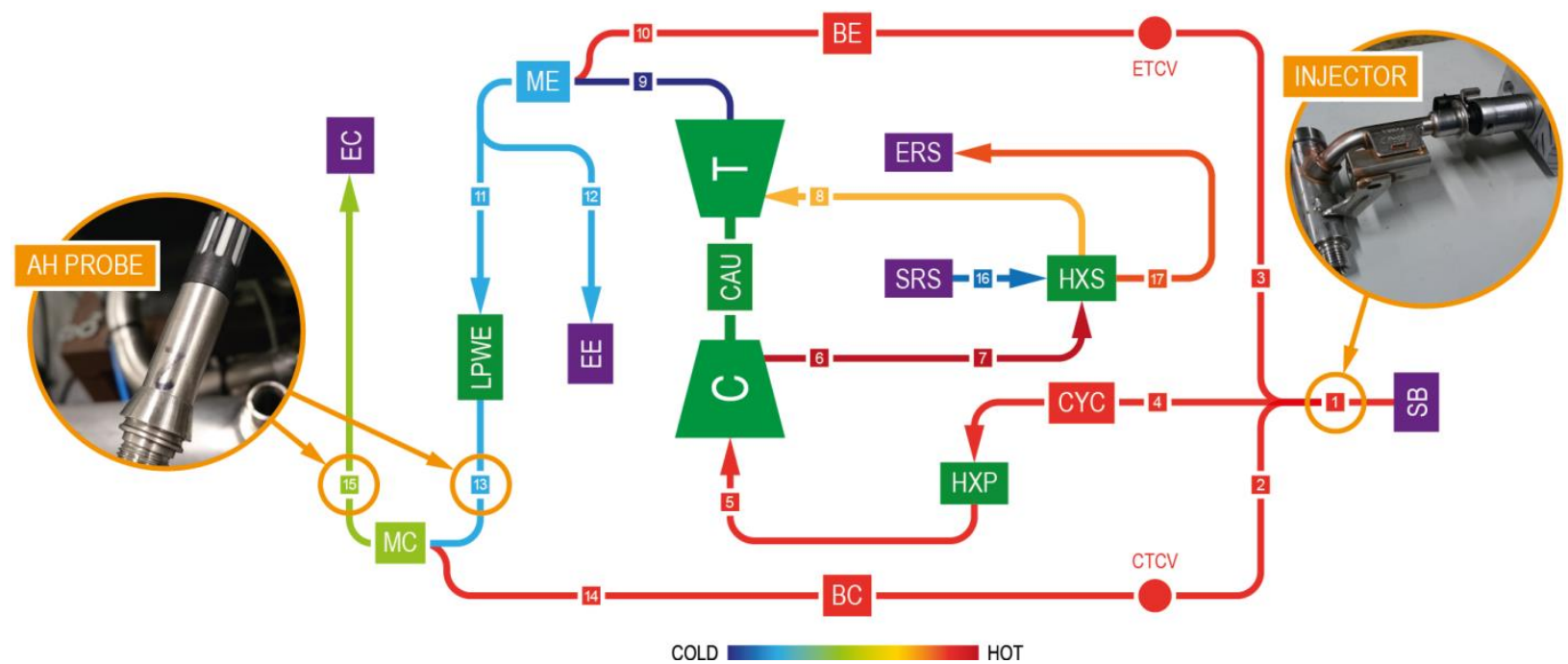

Figure 2. ECS Schematic Instrumentation locations are numbered, thermodynamic components are indicated by a flow direction arrow and green block, boundary conditions by a purple block, and system location labels by a block coloured in accordance with the flow path.

A $368 \mathrm{~kW}$ positive displacement compressor provides an air farm of $40 \mathrm{~m} 3$ with dry air at $13.8 \mathrm{Bar}$ for bleed air mass flow rate, which is heated at ECS inlet. High pressure atomised water is injected upstream of the bypass branches, with the use of a Gasoline Direct Injection (GDI) fuel injector supplied with water at 150Bar. The injector 
flow rate is mapped prior to testing and humidity is measured at two locations with hygroscopic polymer capacitance RH sensors. Ram air is provided by a low pressure, high flow rate electric fan situated in the test cell.

The GTF is primarily limited by ram air flow rate supply and has no primary heat exchanger ram air flow. The ram air flow through the secondary heat exchanger generates up to $12 \mathrm{~kW}$ of heat rejection; allowing direct replication of 27 design flight cases from a total of 41 stock conditions. Cold Air Unit (CAU) inlet bleed air conditions can be matched to the full extent of the flight envelope in terms of flow rate, temperature, pressure, and humidity.

\section{A. Performance Metrics}

Coefficient of Performance (CoP) is a common measure of effectiveness for heat pumps and refrigeration cycles. As the primary function of the ECS is to reduce the temperature of the working fluid, CoP finds common use in evaluation of its performance. The general form of $\mathrm{CoP}$ for a refrigeration cycle is given by: ${ }^{10}$

$$
\mathrm{CoP}=\frac{\dot{Q}_{\text {out }}}{\dot{W}_{\text {in }}}=\frac{\text { Net useful heat transfer rate }}{\text { Total work consumption rate }}
$$

This definition is straightforward to apply when considering a traditional closed cycle, such as that used in a phase-change refrigeration unit. A mechanical work term is usually directly calculable (for example current draw by an electric motor), whilst the heat transfer rate is usually based upon the net result of changing or maintaining the temperature of a closed volume.

Where an ECS is concerned, it is less straightforward to define each constituent of the equation. The process of cooling bleed air is not a 'useful output' in itself; when that fluid is then used to remove quantifiable heat loads elsewhere. Meanwhile, the work consumption rate is equally hard to define as no direct mechanical power is supplied to the ECS. All of the fluid streams of interest are open, whereas usually a coolant working fluid would operate in a closed cycle. CoP as a measure of ECS effectiveness has very little to do with the energy cost to the aircraft as a whole.

For this investigation, the method to calculate $\mathrm{CoP}$ is given by:

$$
C_{o P} P_{S y s}=\frac{\dot{Q}_{S y s}}{\dot{W}_{S B}+\dot{W}_{S R}-\dot{W}_{E S y s}} \quad\left[C_{o P} P_{C y c}=\frac{\dot{Q}_{C y c}}{\dot{W}_{S C y c}+\dot{W}_{S R S}-\dot{W}_{E C y c}}\right]
$$

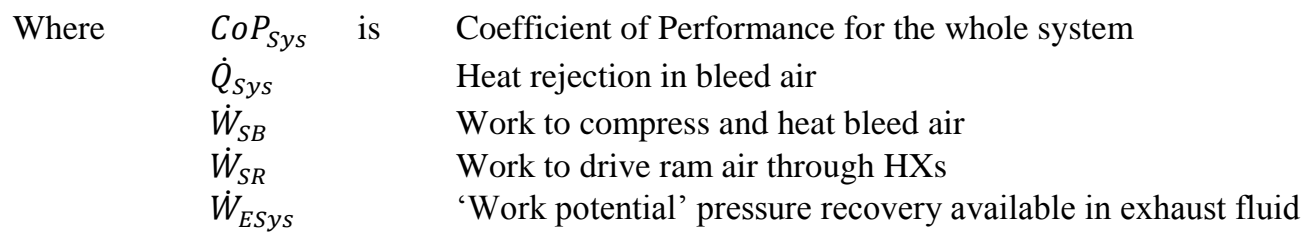

This equation is applied to both the overall ECS at system level and to the cycle at component level $\left(C_{C o P_{C y c}}\right)$. System CoP considers flow between SB and pack exhaust, whereas cycle CoP considers only the flow through CAU and HXS. The exhaust pressure recovery $\left(\dot{W}_{E}\right)$ term is included so that the internal energy remaining in the flow at ECS exhaust is quantified. This allows for the CoP calculation to account for the pressure loss across the system, which varies with bleed air inlet condition and TCV position.

Each of the terms in the CoP equation is calculated by the following methods. There is a desire to idealise the calculations as much as possible, so that any irreversibility effects associated with the process used to actually perform the work on the bleed air flow external to the ECS are neglected:

$$
\begin{gathered}
\{1\}: Q_{S y s}, Q_{C y c}: Q=\dot{m} \Delta h=\dot{m} C_{P} \Delta T \\
\{2\}: \dot{W}_{S B}, \dot{W}_{S C y c}, W_{E S y s}, W_{E C y c}: \dot{W}=\dot{m} C_{P} T_{A}\left[\left(\frac{P}{P_{A}}\right)^{\frac{\gamma-1}{\gamma}}-1\right] \\
\{3\}: \dot{W}_{S R}, \dot{W}_{S R S}: \dot{W}=\frac{\Delta P_{R} \dot{m}}{\rho}=\frac{\Delta P_{R} \dot{m} R T_{S R}}{P_{S R}}
\end{gathered}
$$

The temperature and pressure ranges seen throughout the cycle allow for air to be approximated as a perfect gas. ${ }^{11}$ The derivation of each equation above is taken from the following assumptions: 
1. From the standard form of steady flow energy equation, neglecting changes in gravitational potential energy and fluid velocity where mechanical work done on or by the fluid is nil. ${ }^{11}$

2. From work done during isentropic compression for an open system. ${ }^{11,12}$ The same equation is used to quantify the exhaust fluid pressure recovery, by assuming that this is the maximum amount of work recoverable from the fluid during isentropic expansion.

3. From the power required to move an item with a force at a velocity, $P=F v$. The net force on the heat exchanger is easy to calculate accurately, given a significant pressure differential in the ram air stream.

\section{B. Humidity Calculations}

The calculation of $\mathrm{AH}$ at system inlet is a function of bleed air mass flow measurement and water injection rate. The difficulty of humidity analysis is the measurement of water content in the processed fluid at pack exhaust and prediction of state of the water content throughout the cycle. ${ }^{13-15}$ Volumetric flow rate of condensate (from WE drain and supply branches) is measured cumulatively over the duration of a test, while Relative Humidity (RH) of the gas at system exhaust is measured in real-time.

The majority of the analysis conducted in this investigation focusses on the effect of humidity on the operation of the system, in terms of exhaust temperature and CoP. In order to further understand the reasons for certain system behaviours, it is desirable to predict the state of the moisture content at each location throughout the system.

The following equations allow the calculation of $\mathrm{RH}$ and condensate level from known $\mathrm{AH}$, measured total mixture pressure, and measured temperature. Basic humidity relationships are given by: ${ }^{13}$

$$
R H=\frac{e_{V}}{e_{S}} \quad A H=\frac{\rho_{M} e_{V}}{R_{V} T}\left[\frac{k g \text { water }}{k g \text { dry air }}\right]
$$

$\begin{array}{llll}\text { Where } & e_{V} & \text { is } & \text { Absolute partial vapour pressure } \\ & e_{S} & & \text { Saturation vapour pressure } \\ \rho_{M} & & \text { Mixture density } \\ R_{V} & & \text { Vapour ideal gas constant }\end{array}$

An empirical relationship for saturation vapour pressure is given by: ${ }^{14}$

$$
e_{S}=C e^{\left[\frac{A t}{B+t}\right]}
$$

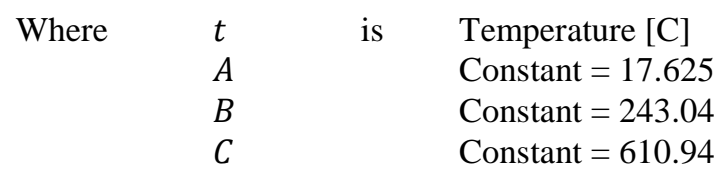

The fluid properties for a mixture of air and water vapour are given by: (adapted from Gibbs-Dalton Law ${ }^{11}$ )

$$
\begin{array}{cl}
\rho_{M}=\frac{P_{M}}{R_{M} T}=\frac{P_{M}(1+A H)}{R_{A} T}\left[1+\frac{A H R_{V}}{R_{A}}\right]^{-1} \quad & \rightarrow \quad R_{M}=\frac{R_{A}+A H R_{V}}{1+A H} \\
h_{M}=h_{A}+A H h_{V}=C_{P_{M}} \Delta T \quad \rightarrow \quad & C_{P_{M}}=C_{P_{A}}+A H C_{P_{V}}
\end{array}
$$

Where $\quad P_{M} \quad$ is $\quad$ Mixture pressure (measured)

When RH > 1, condensate level is given by the difference between the actual $\mathrm{AH}$ and the $\mathrm{AH}$ that would be required to saturate the flow:

$$
A H_{C}=A H-A H_{S} \quad A H_{S}=\frac{\rho_{M} e_{S}}{R_{V} T}
$$

Where $A H_{C}$ is Condensate ratio $[\mathrm{kg} / \mathrm{kg}]$

$A H_{S} \quad$ Absolute humidity at saturation $[\mathrm{kg} / \mathrm{kg}]$ 


\section{Results \& Discussion}

The results of several tests are presented below. Observations from each figure are analysed immediately below in list form, whilst in-depth discussions of the resultant phenomena appear in prose. The tests conducted analyse the ECS in two ways. Thermodynamic analysis explains how the variation in bleed air AH affects the performance of the ECS both at system- and component- level. Tests are conducted which further the understanding of LPWE, and the effect this method of water control has on the operation of the ECS.

\section{A. Thermodynamic Analysis of Working Fluid}

The analysis presented below considers the behaviour of the ECS with respect to working fluid variation. The controlled variable is $\mathrm{AH}$, and the range of test conditions reflects the design limits of the donor aircraft. All tests conducted are representative of operational conditions.

\section{Overall System Performance}

For a bleed air inlet absolute humidity sweep at constant temperature and pressure, Figure 3 below depicts turbine-out temperature (T9) and CoP for both the entire system and the cycle in isolation. This is a typical system response to variation in bleed air $\mathrm{AH}$, with all other controls kept constant. The effects on the performance of the system are purely as a result of the moisture content present in the working fluid.

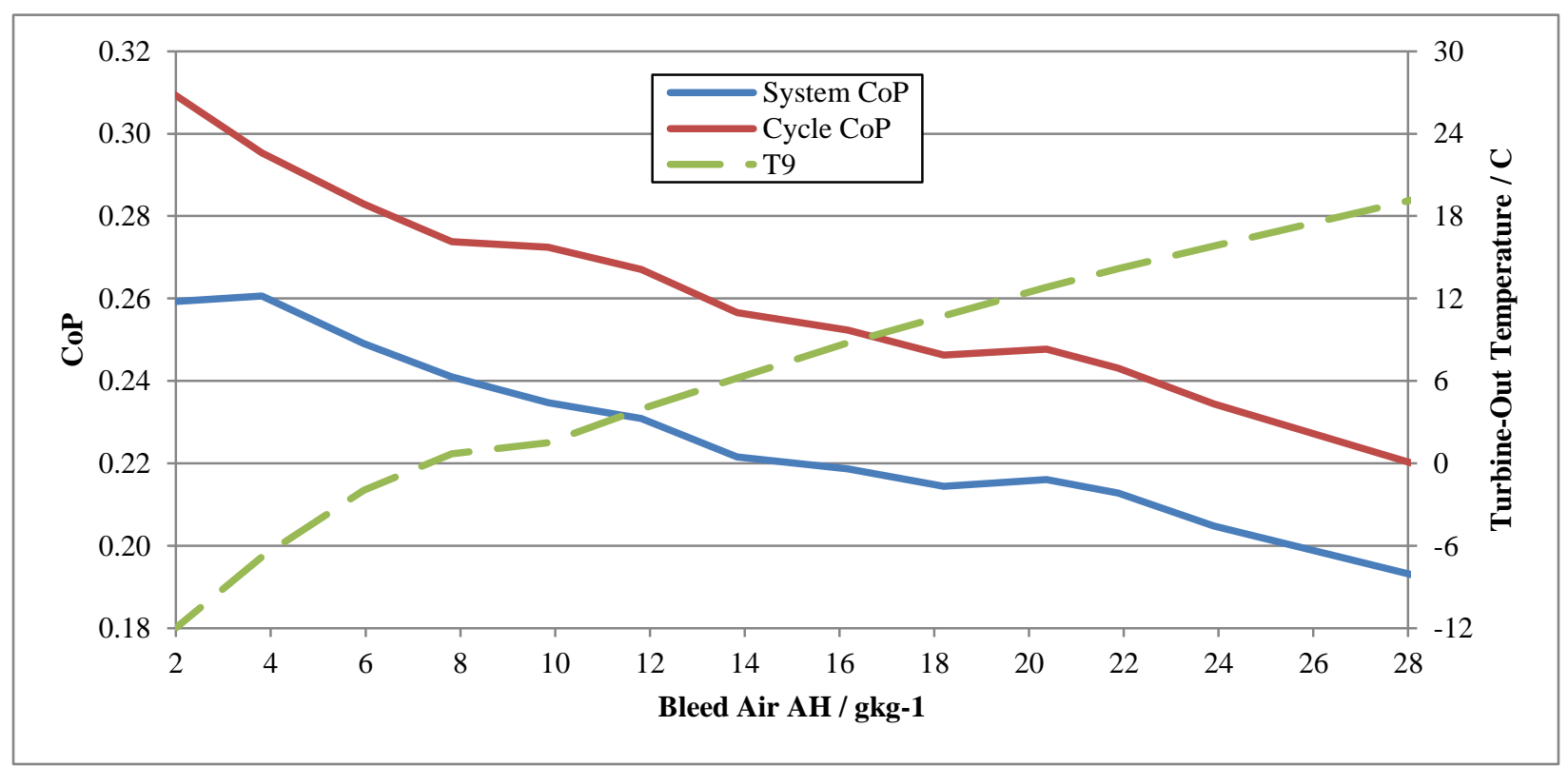

Figure 3. CoP and Turbine-Out Temperature against Bleed Air AH. $\quad$ CoP decrease with increasing AH is approximately linear, whilst turbine-out temperature increase is both significant in magnitude and distinctly non-linear.

- As inlet humidity increases, CoP decreases for both the cycle and the entire system. At low inlet humidity, the two metrics diverge due to low turbine-out temperatures driving heat flow from ambient through the walls of the pipe run between turbine-out and WE-in. This causes an increase in temperature between turbine-out and pack exhaust that degrades system CoP.

- Cycle CoP is greater than system CoP for two reasons: bleed air heat rejection is greater due to the increase in temperature between turbine-out and pack exhaust; and less power is required due to pressure loss in HXP, bypass branches, and WE.

- Turbine-out temperature increases significantly with increasing inlet humidity. A plateau witnessed at $0 \mathrm{C}$ separates two distinct linear regions of operation.

- Single-phase flow in the turbine can be assumed at low inlet humidity, whilst the flow is two-phase at high humidity. This behaviour is not reflected in CoP.

- The relative magnitudes of degradation witnessed in the two performance metrics are dissimilar: across the range of inlet humidity tested, CoP degrades by $27 \%$ whilst turbine-out temperature degrades by $35 \mathrm{C}$. Equipment delivery temperature is regulated by design between $0-4 \mathrm{C}$; this variation in turbine-out temperature represents 8.75 times the design spec limit for equipment delivery temperature. 
This test serves to illustrate the inability of a first law calculation to fully explain how the system responds to an increase in bleed air absolute humidity. Whilst a fall in CoP of $27 \%$ is significant, the system sees similar magnitudes of CoP degradation for operational variations in bleed air supply temperature and pressure. These variations however, do not degrade the ability of the system to deliver low turbine-out and pack exhaust temperatures to the same degree as with increasing absolute humidity.

Typically, CoP is a governing metric for system design and indication of operational efficiency. However, the pilot and avionics measure the performance of the ECS by delivery temperature rather than energy conservation.

\section{Analysis of Coefficient of Performance}

Figure 4 below shows how the constituents of CoP vary for a similar test as shown in Figure 3, as a means of analysing where system performance is lost.

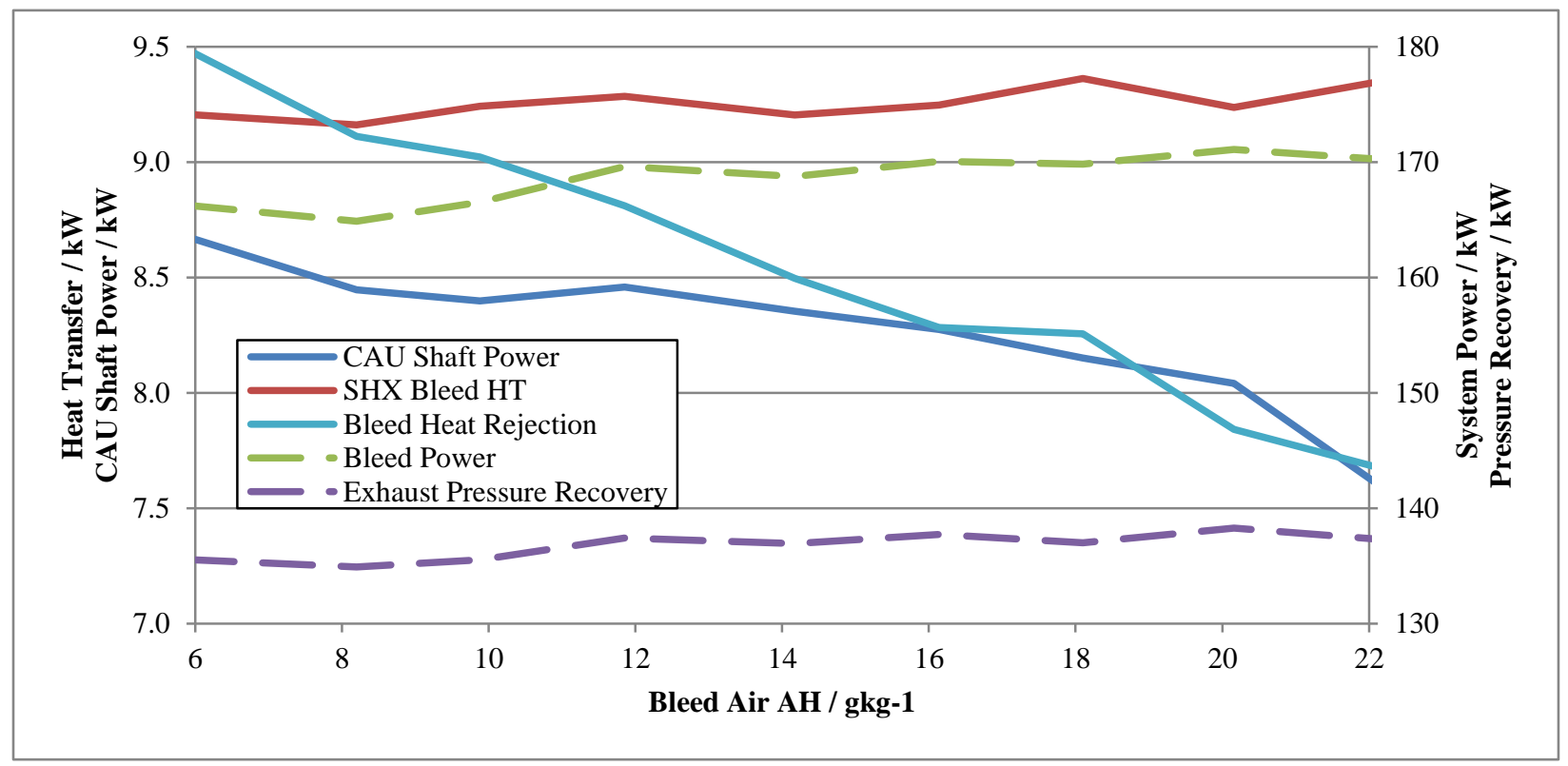

Figure 4. Components of System CoP against Bleed Air AH. System power and HXS heat rejection remain constant whilst total bleed air heat rejection and CAU shaft power fall linearly, for increasing AH.

- $\quad$ System CoP degrades linearly with increasing bleed air inlet AH. Both CoP denominator terms (bleed air power and exhaust pressure recovery) remain approximately constant irrespective of bleed air AH.

- HXS heat rejection remains constant throughout. During constant AH operation, this property is found to exhibit a strong linear relationship with system CoP. ${ }^{9,16}$

- The reduction in bleed air heat rejection causes the observed reduction in CoP, through reduction in $\mathrm{CAU}$ shaft power. Across the range of inlet humidity tested; CAU shaft speed falls from $67 \mathrm{krpm}$ to $60 \mathrm{krpm}$, along with a drop of $3.5 \%$ in both compressor and turbine efficiencies.

- The ability of the CAU to extract energy (shaft power) from bleed air is reduced as humidity increases, for two reasons:

○ Degradation of working fluid thermodynamic properties (density).

- Phase change of water content in the turbine (discussed in more detail in Section IIIA.4).

Increasing bleed air humidity does not markedly change the heat transfer rate in HXS or the power requirement of the system. Therefore, the degradation of system performance with increasing bleed air inlet humidity can be traced almost entirely to CAU: specifically the turbine. The performance of the entire system is driven by the amount of bleed air energy successfully extracted by the turbine.

\section{Analysis of Working Fluid Thermodynamic Properties}

The energy-conservation principle calculations performed on the system are heavily influenced by the density and specific heat capacity of the working fluid. The thermal conductivity of the fluid is influenced by both of these properties, driving differences in convective heat transfer performance. Specific heat capacity describes the change 


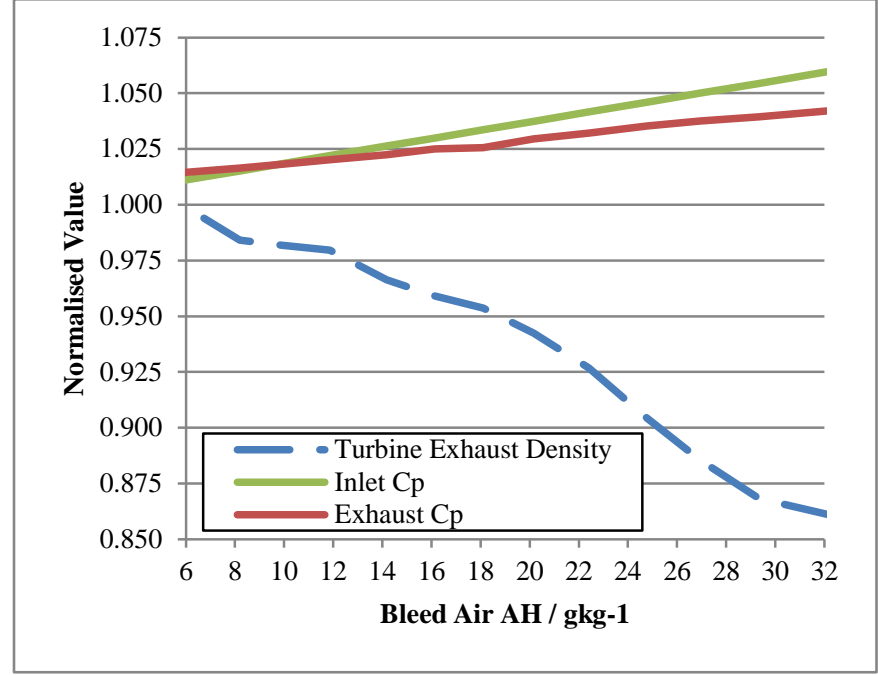

Figure 5. Normalised Specific Heat Capacity and Density against Bleed Air AH.

in temperature for a given heat flow rate, where a higher specific heat capacity facilitates a higher heat flow rate for a given temperature differential. A reduction in density increases relative humidity for a given absolute humidity, due to an increase in vapour pressure (increasing the tendency of the water content to condense in the turbine).

Figure 5 shows how these parameters vary with bleed air inlet absolute humidity. All parameters are normalised for their standard 'dry' values.

- $\quad$ Small increases are found in specific heat capacity with increasing humidity. This serves to increase the amount of heat transfer possible in the system for a constant temperature differential, increasing CoP.

- $\quad$ Significant decreases are found in working fluid density with increasing humidity. This is due to the difference in molar mass between water vapour $(\mathrm{H} 2 \mathrm{O})$ and the common constituents of air that it displaces $(\mathrm{N} 2, \mathrm{O} 2)$.

- The shape of the density curve closely mirrors that of cycle CoP (Figure 3), indicating a close relationship between the first law efficiency calculation and working fluid characteristics.

\section{Analysis of Turbine Phase Change Conditions}

Figure 6 below shows how RH varies throughout the system for constant bleed air inlet AH and varying inlet pressure. The calculation of $\mathrm{RH}$ accounts for both temperature and pressure effects, where values greater than 1 indicate that the working fluid is saturated and condensate is present.

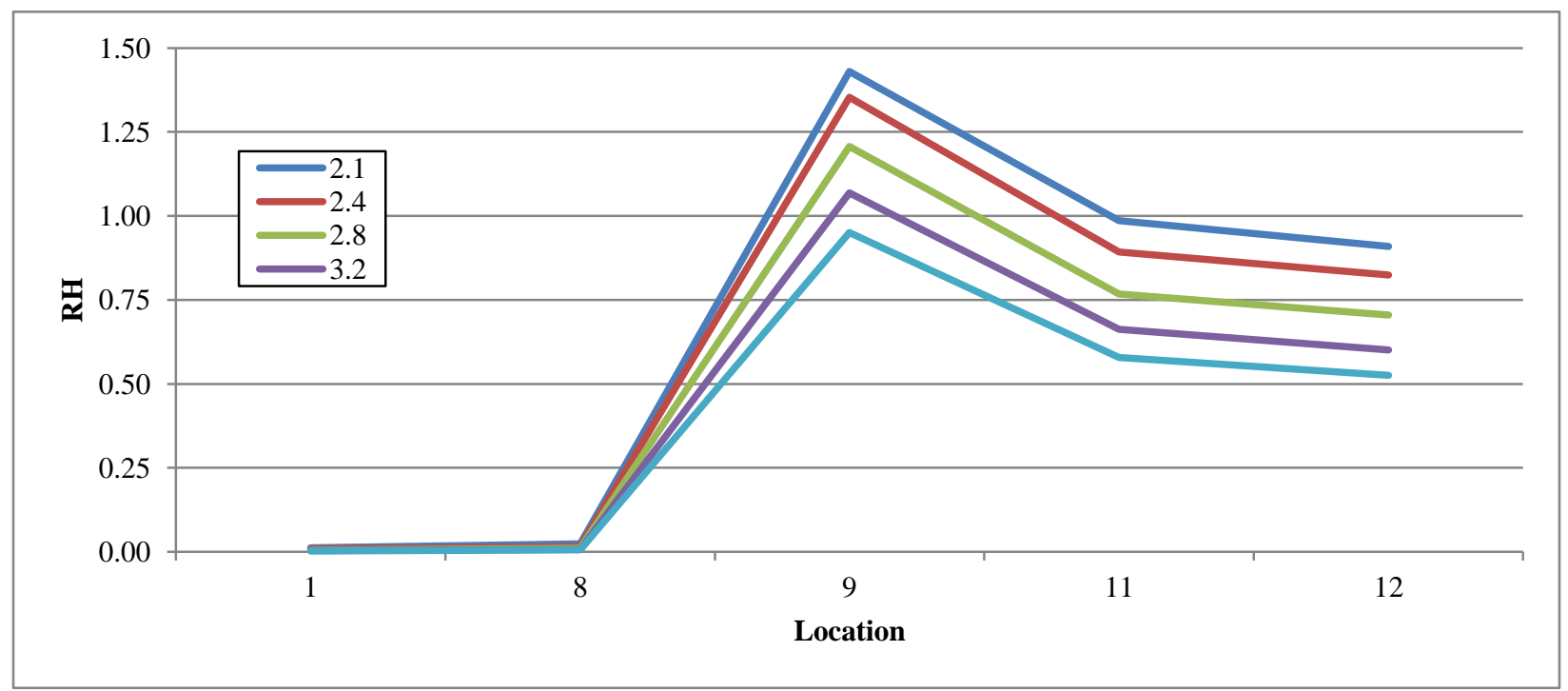

Figure 6. RH Variation through ECS. other parameters being equal.

Where each curve represents a different bleed air pressure (2.1-3.2 Bar), all

- $\quad$ Relative humidity at high temperature (system inlet / up to turbine-out) is universally low.

- An increase in bleed air pressure drives greater bleed air mass flow, inducing higher levels of irreversibility in the air cycle through turbulence and energy dissipation. This increases both temperature and pressure at turbine exhaust. Increasing temperature and pressure, when $\mathrm{AH}$ is fixed, reduces $\mathrm{RH}$. 
- As operating conditions drive relative humidity at turbine exhaust towards 1 , vapour pressure exceeds saturation pressure which causes condensate to form. At the lowest inlet pressure state tested (blue curve, Figure 6), the condensate formed at the turbine exhaust is calculated to be approx. $4.2 \mathrm{~g}$ per kg bleed air.

This analysis indicates how the effect of working fluid moisture content on the performance of the system is not limited to the quantity of moisture present. The operating condition of the ECS affects the rate of condensate generation for a fixed absolute moisture content.

The formation of condensate is a requirement for water extraction, as gaseous water cannot be separated from air by method of coalescing sock and centrifuge. In order for this design to work, condensation must occur in the turbine; which as shown degrades the performance of the entire cycle. The system has no mechanism in place to recover the energy released during the formation of condensate; rather it is dissipated internally to friction.

\section{B. Low-Pressure Water Extraction Investigation}

Figure 7 below shows relative humidity and absolute condensate for turbine-out (9), WE-in (11), and equipment feed (12): the critical regions for water extraction efficiency analysis. The ECS inlet conditions are of low temperature and pressure, so that the resultant low turbine-out temperature drives high relative humidity and condensate content for a given absolute humidity.

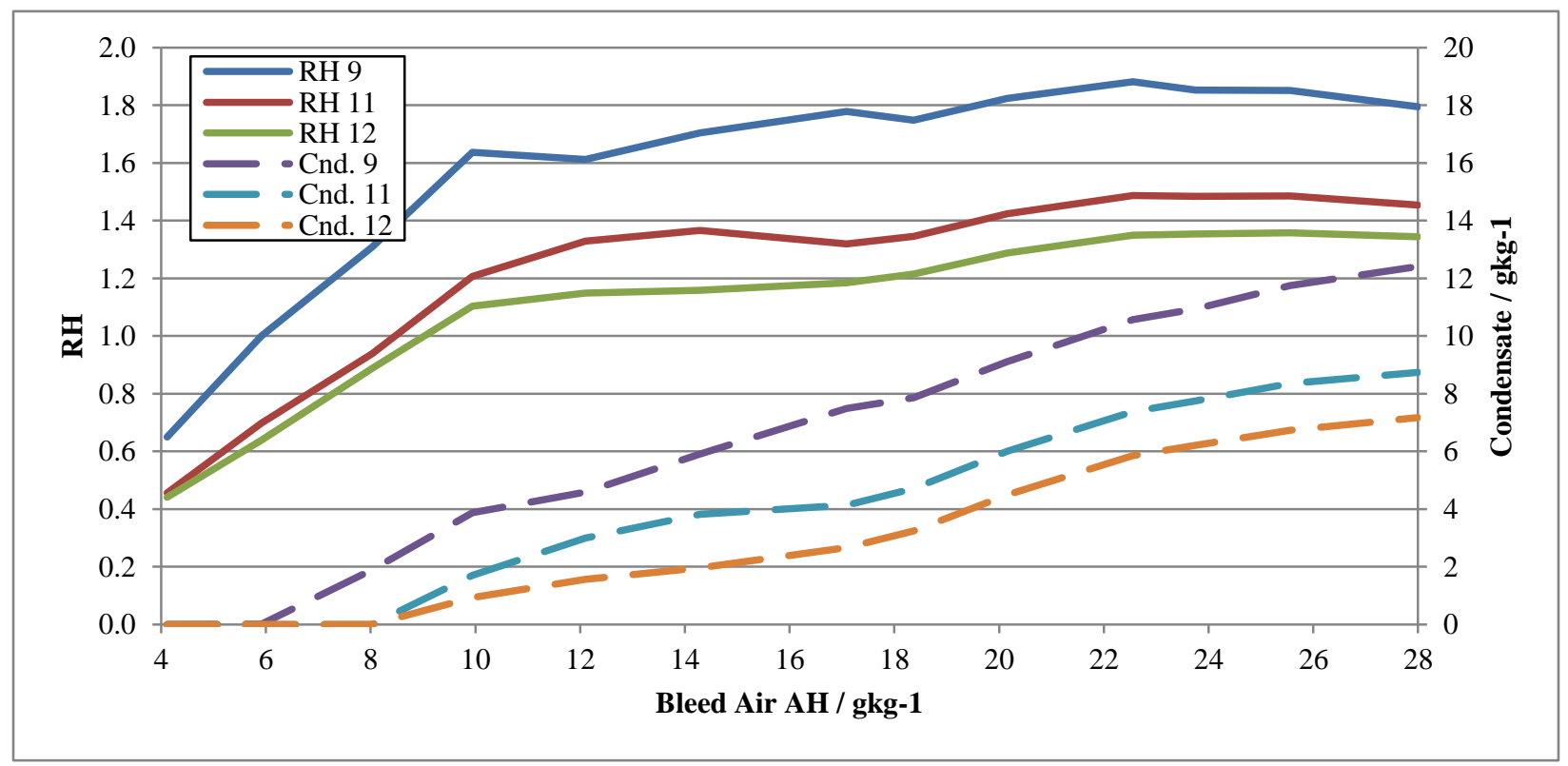

Figure 7. RH and Condensate against Bleed Air AH.

- The difference between the amount of condensate present at turbine-out and WE-in is resultant of revapourisation of condensate. The energy required for vapourisation is supplied by the temperature differential between turbine-out and ambient. As a result, the location of the water extractor is critical.

- The equipment feed take-off (EE) is prior to WE inlet, and therefore it is highly likely that saturated air is delivered to the avionics at the design temperatures.

In order for the water extractor to perform well at this location, significant amounts of condensate must be generated in the turbine and carried through to the coalescing sock. Phase change in the turbine reduces its capacity to generate low turbine-out temperatures. The system design goals of bleed air water extraction and low turbine-out temperatures are at odds with each other; in order to extract any water from the bleed air, the performance of the cycle must be degraded.

Figure 8 below shows physical measurements of the condensate collected at the water extractor drain and cabin supply branch, against bleed air inlet humidity. Also plotted is calculated condensate at turbine exhaust and BE mass flow. The test was conducted over a period of approximately one hour with automatic valve position control. 


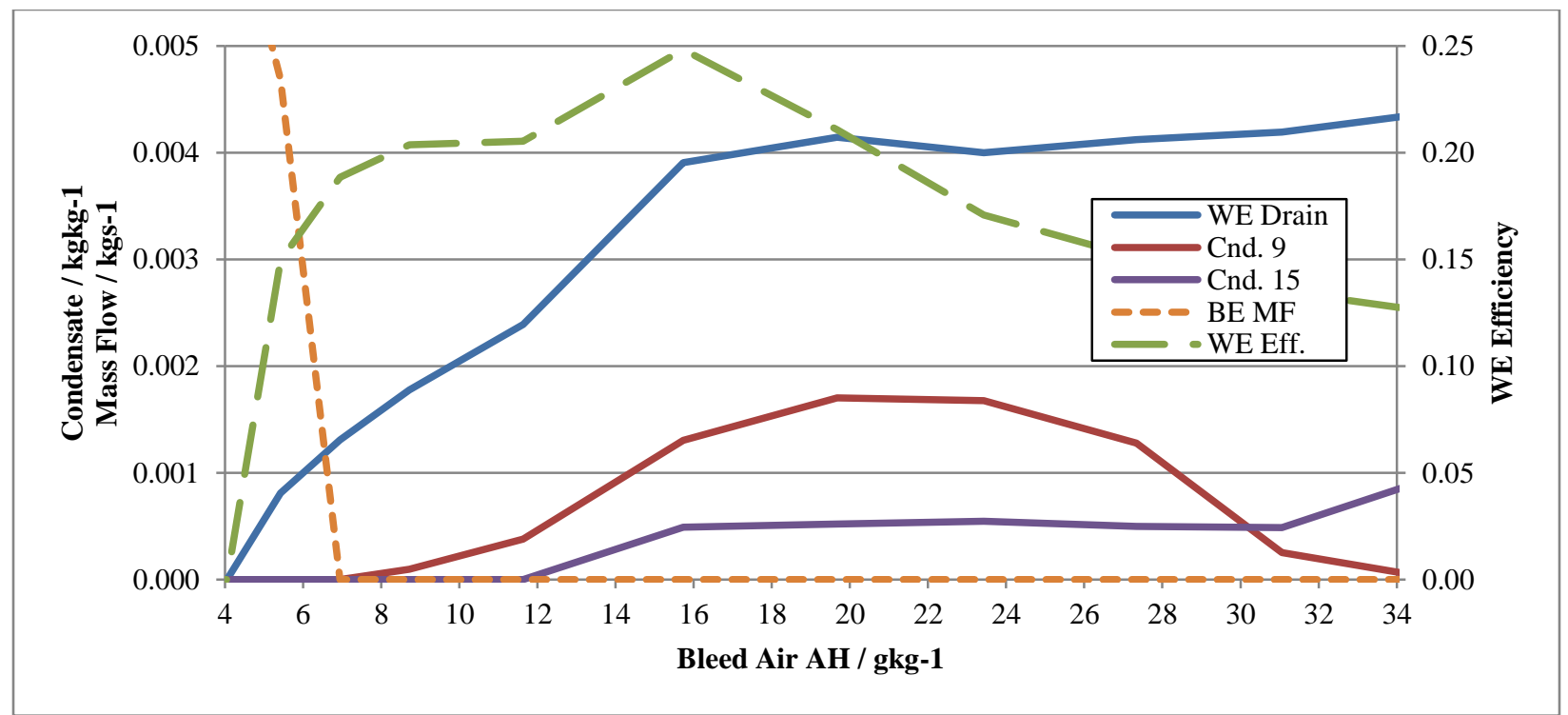

Figure 8. Various System and Water Extractor Parameters against Bleed Air AH.

Automatic TCV regulation shuts ETCV at approx. $7 \mathrm{gkg}-1 \mathrm{AH}$, whilst the condensate extracted from WE does not exceed a peak found at approx. $15 \mathrm{gkg}-1$ AH.

- Bypass mass flow is reduced with increasing inlet humidity due to the reduced capacity of the air cycle to generate low turbine-out temperatures. ETCV shuts at approximately $7 \mathrm{gkg}-1$ absolute inlet humidity, which coincides with the point at which condensate starts to form at turbine exhaust.

- Throughout this test, the calculated relative humidity at water extractor inlet is less than 1 . Whilst this indicates that no condensate should be present (hence its omission from the graph), this is proven false by the fact that condensate is extracted from the water extractor drain when bleed air inlet humidity exceeds approximately $4 \mathrm{gkg}-1$.

- When considering total water content in the bleed air (at any state or phase), water extraction efficiency of the system peaks at around $25 \%$, at $15 \mathrm{gkg}-1$ absolute inlet humidity for this test. After this point, condensate extraction is approximately constant for increasing inlet humidity, and water extraction efficiency falls appropriately.

- Condensate is present in the cabin feed at moderate-to-high levels of inlet absolute humidity. This is due to the (relatively) low temperature of the pack exhaust and its correspondingly low saturation vapour pressure, as well as poor water extraction efficiency. The presence of condensate at this location confirms that the cabin delivery air is saturated under certain circumstances (i.e. high inlet humidity, low cabin temperature demand, low turbine-out temperature).

- Turbine-out condensate falls off at high levels of inlet humidity; due to high turbine-out temperature, which is resultant of phase-change in the turbine, which is in turn resultant of high inlet humidity. This serves as a reminder of the ability of a bootstrap air cycle machine to self-regulate.

A key reason for poor water extraction efficiency is the addition of bypass airflow prior to WE inlet. The addition of hot air at this location is primarily for the purpose of component protection, controlling WE inlet (and equipment feed) between $0^{\circ} \mathrm{C}-4^{\circ} \mathrm{C}$ to avoid blockage of the coalescing sock through icing. However, as a function of increasing the temperature of this flow, the bypass air also acts to re-vaporise condensate before the WE inlet. This behaviour is seen because condensate is only generated at the turbine exhaust when the ETCV is shut (7gkg-1). The net result is a reduction in WE inlet condensate, reducing the efficiency of water extraction purely by design of ECS pipework.

Throughout the test, the water extractor appears to generate rather than remove condensate. In reality this is incorrect, and a function of the process by which absolute condensate is measured (Cnd. 15) or calculated (Cnd. 9). The temperature gradient between the centre of the wheel of the turbine (where phase change takes place) and WE inlet is steep; therefore the measurement location has a strong influence on the calculated levels of humidity present. This explains why the calculated level of condensate present at turbine-out is less than the amount of condensate collected from the water extractor drain. 
The saturation calculation tends to single phase flow at WE inlet during high AH conditions as a high turbineout temperature allows an increase in absolute moisture content before saturation. However, as the flow velocity at turbine-out is high (approx. 45ms-1) and the pipe run between turbine and WE is relatively short (approx. $0.3 \mathrm{~m}$ ) there is insufficient time for the water content to completely re-vaporise prior to the WE. It was found in other testing at lower bleed air mass flow levels (lower flow velocity) the measured water extraction was much closer to calculated levels. This indicates that complex 3-D fluid flow structures are integral to both water extractor efficiency and calculation accuracy.

The fall-off in water extraction efficiency witnessed in Figure 8 is also partially attributed to the duration of the test and resultant saturation of the coalescing sock in WE. As the WE system saturates, the back pressure on the air cycle increases. This reduces the Total Pressure Ratio of the Cycle (TCPR), reducing its ability to extract internal energy from the bleed air. Both system CoP and turbine-out temperature are highly dependent on TCPR - therefore restricted performance during times of WE saturation can not only be attributed to the aforementioned poorer working fluid properties.

Once the coalescing sock in WE is saturated, the dynamics of the system are too complex to confidently apply steady-state analysis methods. The PRV opens once the pressure at WE-in increases past a threshold value (which happens over a period of up to 40 minutes). TCPR then increases, and the performance of the cycle improves. This results in different dynamics of the water content at turbine-out, and drives a change in the flow structure in WE. The PRV has many complex dynamics of its own, primarily due to limited damping, which influences the rate at which this cycle repeats.

The performance of the system is highly dependent on the operating condition immediately prior to the present, when reasonable levels of inlet humidity exist. The system never truly reaches a stable equilibrium. Modelling this behaviour accurately would require extensive knowledge of both the flow structure in the system and of the system's operating conditions for a significant amount of time prior to the modelled state. This is not the case when operating with dry air, and highlights how system design without consideration of transient effects or a good understanding of humidity behaviour can lead to unexpected results. The differential between calculated and measured data is a validation of the requirement for experimental assessment of working fluid on ECS performance and an explanation to the inaccuracies of modelling these transient processes through saturation calculations.

\section{Transient Operational Effects}

One particularly drastic example of WE transient effects can be witnessed when operating with high bleed air inlet humidity and sub-zero WE-in temperature. Condensate forms as a fog in the turbine and coalesces in WE as usual. However due to the low temperature, the condensate freezes and therefore cannot drain. This leads to a significant blockage in WE until the PRV opens and ice is discharged from the system. Figure 9 below depicts a test where these conditions were sustained for 20 minutes.

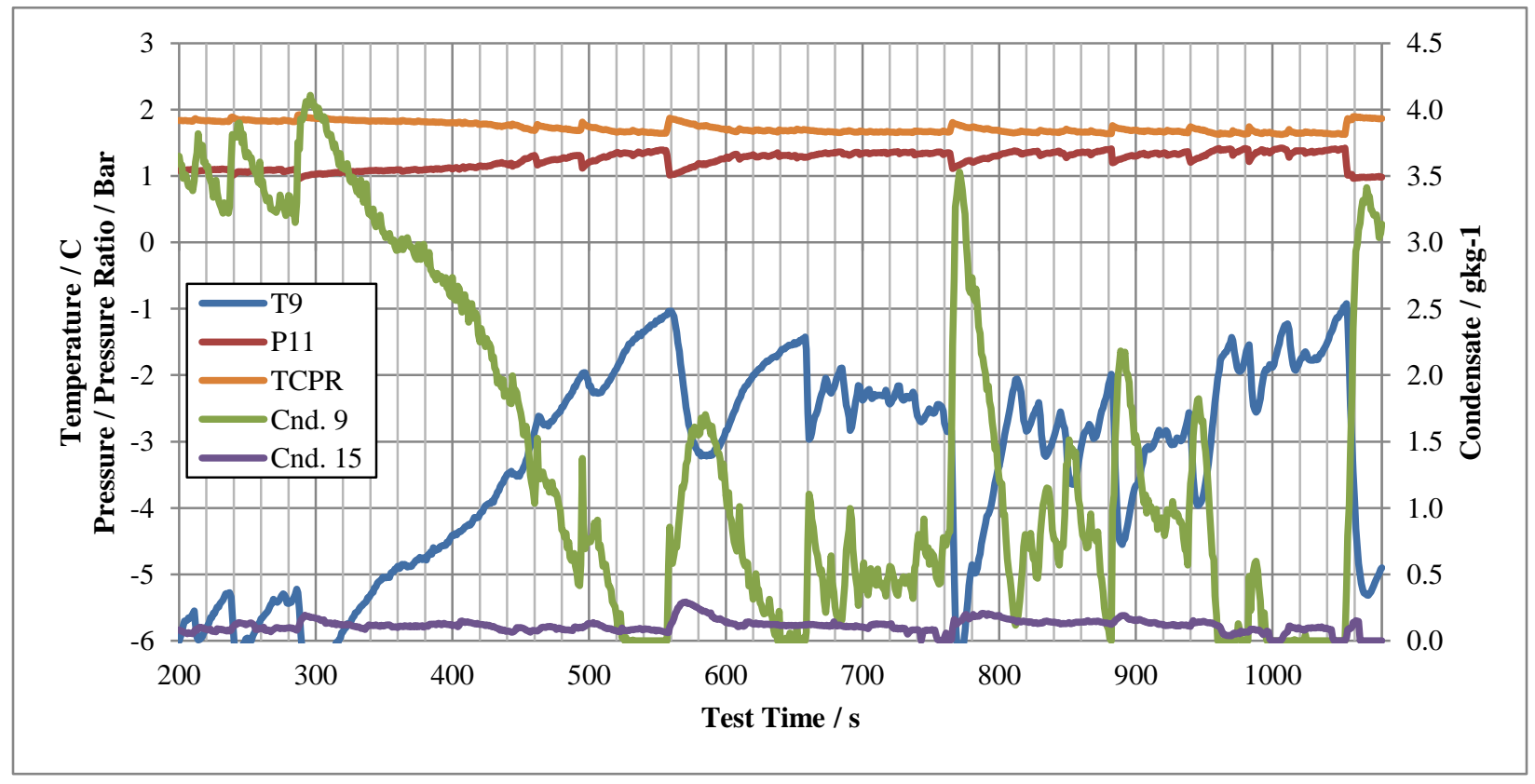

Figure 9. System Behaviour during Transient Icing Conditions. 
- Turbine-out temperature is maintained sub-zero for the duration of the test; whilst relative humidity at this location is consistently above 1 . This allows condensate to freeze and build-up in WE.

- WE blockage increases consistently from 200-560s. Turbine and cycle pressure ratios decrease, whilst WEin pressure and turbine-out temperature increase. The PRV can be seen to 'crack' 2-3 times throughout this period.

- WE blockage is relatively constant from 560-1050s. The system finds a near-equilibrium state for this period. System performance degradation causes elevated turbine-out temperature and reduced condensate formation.

- During this period, the PRV opens several times. This allows small amounts of ice and snow to be discharged into the cabin feed. The performance of the system increases when the PRV opens, turbine-out temperature falls, more frozen condensate is formed to replace that which was previously discharged.

- At 1050s, the volume between the coalescing sock and the exhaust of the water extractor blocks completely and the PRV opens fully. This allows the majority of the frozen condensate (of significant mass) stored in WE to rapidly discharge into the cabin feed. The test is aborted at this point in order to inspect the interior of WE.

Figure 10 below shows the interior of WE and the coalescing sock directly after the test was aborted. Significant amounts of frozen condensate remain in the component even after the majority is discharged from the system. It is thought that the entire volume between the downstream side of the coalescing sock and the swirl vanes at the exhaust of the water extractor fills with snow and ice.

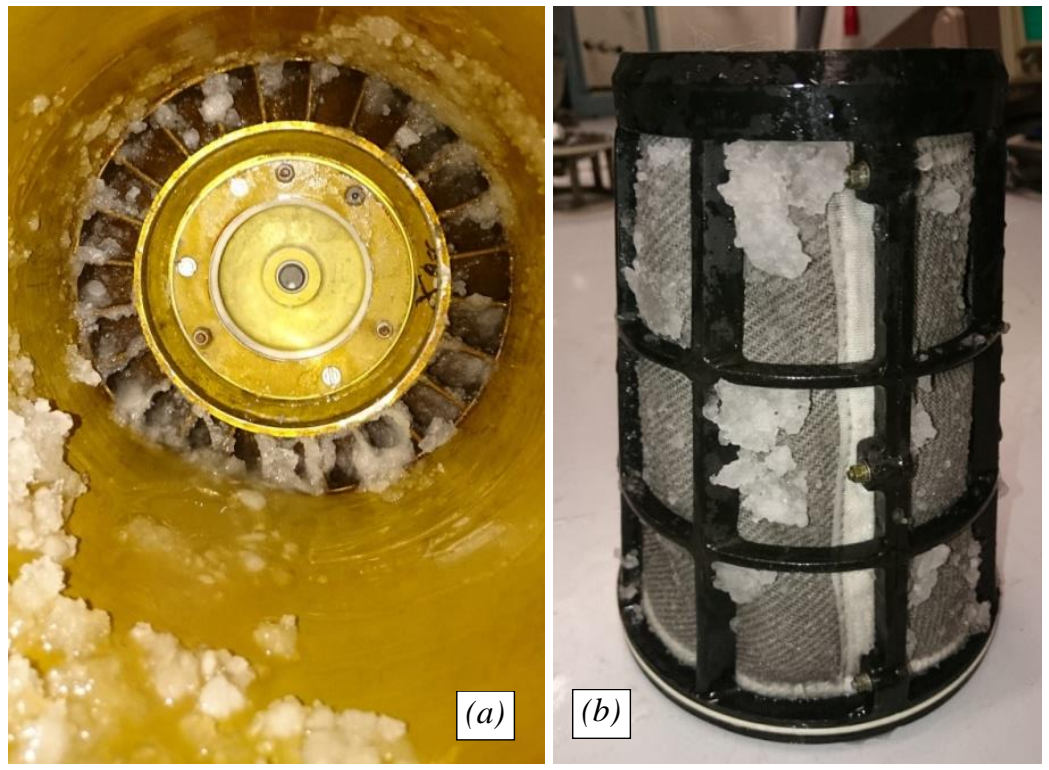

Figure 10. Water Extractor after Transient Icing Test. (a) shows the interior of WE, (b) shows the outside of the coalescing sock.

\section{Conclusions}

The performance of a typical bootstrap air cycle machine degrades with increasing working fluid absolute humidity. This is fundamentally for two reasons:

- Reduction of working fluid density leads to reduced bleed air heat rejection capability of the system. The effects of this outweigh those of increased specific heat capacity and thermal conductivity seen with increased moisture content.

- Under certain conditions, the exothermic process of phase change in the turbine limits the amount of useful work it may produce. The net result is reduced shaft power, increased turbine-out temperature, and degradation of system performance. 
Working fluid properties are the main driver of system energy efficiency $(\mathrm{CoP})$ for a given architecture, irrespective of phase change. However, an inability to recover the energy released by phase change in the turbine degrades the cooling capacity of the cycle by increasing pack exhaust temperature. As energy is conserved throughout, calculated first-law efficiency is independent of phase change. During operation, thermal management systems are concerned with air delivery temperature and mass flow rate over energy efficiency.

A system that utilises LPWE requires a phase change event in the turbine in order to extract any water from the working fluid. This introduces the concept of a trade-off between cooling capacity and minimised exhaust condensate content. Cooling capacity degradation is a function of water separation at the turbine, rather than a control of water extractor inlet conditions.

At high AH a number of time and flow dependent transient behaviours related to water extraction efficiency and blocking are witnessed. Although the behaviour is localised to component level (saturation of LPWS coalescing sock), a variation in system level thermodynamic performance is measured. This is difficult to predict or calculate as it is the function of complex 3D flows and time dependent saturation of the water extractor coalescing sock. These transient behaviours highlight the difficultly and potential inaccuracies of a purely modelling based study of ECS response to absolute inlet humidity.

In real world LPWE implementation, bleed air bypass is used to maintain WE inlet conditions above 0C. Whilst the intent of this control is component protection (from frozen condensate), ultimately it masks the true effect of moisture content in the air cycle.

Total water extraction efficiency is found to be no more than $25 \%$. Poor water condensate control of LPWE ECS is as a result of the decoupling of separation and extraction processes. The physical distance between these two processes facilitates secondary uncontrolled phase change events. The re-vapourisation of condensate is due to both the addition of bypass air and heat transfer from ambient. The net result is reduced condensate levels at WE inlet for the same AH and cost to the cycle of condensate formation.

High levels of bleed air inlet humidity are only found at low altitude in high temperature environments, reducing the cooling capacity of the system through performance degradation. The requirement of low turbine exhaust temperatures is most prevalent at these conditions. Typically, heat rejection is high due to additional kinetic thermal loading from the ambient environment. Additionally, ram air flow rates and available power are often low, for example: during ground operations and idle descent. The compounding of these factors creates a worst case scenario for the ECS.

The thermodynamic response to the implementation of high pressure water separation is the ability to achieve low turbine-out temperatures with high bleed air moisture content. Control of the phase change events in the high pressure region allows conditioning of the working fluid at turbine inlet for significantly improved expansion efficiency.

\section{Acknowledgements}

This project is co-funded by: the Engineering and Physical Sciences Research Council (EPSRC) UK, BAE Systems, and Loughborough University. The authors would like to thank the engineering staff at BAE Systems Warton and Brough for their support and guidance.

\section{Bibliography}

1 Matullch, D., "High-Temperature Bootstrap Compared with F15 Growth Air Cycle Air Conditioning System," 1989.

2 Strattan, L. A., "Capabilities and Limitations of Air Cooled Avionic Packages," 1983, 10.4271/831105.

3 Department of Defence, MIL-STD-810G - Environmental Engineering Considerations and Laboratory Tests, 2008 .

4 Fourquet, H., Taillepied, D., Novo, N., and Rolland, A., "Performance Validation of a New Water Separator Concept," 2000.

5 Jones, A. B., Childs, T., Chen, R., and Murray, A., "Thermal Sensitivity Analysis of Avionic and Environmental Control Subsystems to Variations in Flight Condition," 54th AIAA Aerospace Sciences Meeting, San Diego, CA: American Institute of Aeronautics and Astronautics, 2016, pp. 1-15.

6 Defrancesco, G. L., "Condensing Cycle Air Conditioning System," 2011.

7 Ryan, S. K., F-15 Environment Control System Improvements, SAE International, 1990, 10.4271/901235.

8 Zhao, H., Hou, Y., Zhu, Y., Chen, L., and Chen, S., "Experimental study on the performance of an aircraft environmental control system," Applied Thermal Engineering, vol. 29, Nov. 2009, pp. 3284-3288, 10.1016/j.applthermaleng.2009.05.002. 
9 Conceição, S. T., Zaparoli, E. L., and Turcio, W. H. L., Thermodynamic Study of Aircraft Air Conditioning Air Cycle Machine: 3-wheel × 4-wheel, SAE International, 2007, 10.4271/2007-01-2579.

10 Bejan, A., Advanced Engineering Thermodynamics (Second Ed.), New York: John Wiley \& Sons, 1997, ISBN 0471148806.

11 Rogers, G. F. C., and Mayhew, Y. R., Engineering Thermodynamics: Work and Heat Transfer : SI Units, Longman Scientific \& Technical, 1992, ISBN 0582045665.

12 NASA, "Compressor Thermodynamics," Beginner's Guide to Propulsion Available: https://www.grc.nasa.gov/www/k-12/airplane/compth.html.

13 Spencer-Gregory, H., and Rourke, F., Hygrometry, London: Crosby Lockwood \& Son, Ltd., 1957.

14 Lawrence, M. G., "The Relationship between Relative Humidity and the Dewpoint Temperature in Moist Air: A Simple Conversion and Applications," Bulletin of the American Meteorological Society, vol. 86, Feb. 2005, pp. 225-233, 10.1175/BAMS-86-2-225.

15 Cramer, O., "The variation of the specific heat ratio and the speed of sound in air with temperature, pressure, humidity, and CO2 concentration," The Journal of the Acoustical Society of America, vol. 93, 1993.

16 Childs, T., Jones, A. B., and Chen, R., "Development of a Full Scale Experimental and Simulation Tool for Environmental Control System Optimisation and Fault Detection," 53rd AIAA Aerospace Sciences Meeting, American Institute of Aeronautics and Astronautics, 2015. 